\title{
How Locus of Control Influences Compatibility with Visualization Style
}

\author{
Caroline Ziemkiewicz* \\ Brown University
}

\author{
R. Jordan Crouser ${ }^{\dagger}$ \\ Tufts University
}

\author{
Ashley Rye Yauilla \\ Winthrop University \\ Remco Chang $\|$ \\ Tufts University
}

\author{
Sara L. Su ${ }^{\S}$ \\ Tufts University \\ William Ribarsky ${ }^{\mathbb{q}}$ \\ UNC Charlotte
}

\begin{abstract}
Existing research suggests that individual personality differences are correlated with a user's speed and accuracy in solving problems with different types of complex visualization systems. In this paper, we extend this research by isolating factors in personality traits as well as in the visualizations that could have contributed to the observed correlation. We focus on a personality trait known as "locus of control," which represents a person's tendency to see themselves as controlled by or in control of external events. To isolate variables of the visualization design, we control extraneous factors such as color, interaction, and labeling, and specifically focus on the overall layout style of the visualizations. We conduct a user study with four visualizations that gradually shift from an indentation metaphor to a containment metaphor and compare the participants' speed, accuracy, and preference with their locus of control. Our findings demonstrate that there is indeed a correlation between the two: participants with an internal locus of control perform more poorly with visualizations that employ a containment metaphor, while those with an external locus of control perform well with such visualizations. We discuss a possible explanation for this relationship based in cognitive psychology and propose that these results can be used to better understand how people use visualizations and how to adapt visual analytics design to an individual user's needs.
\end{abstract}

Index Terms: H.5.m [Information Interfaces and Presentation (HCI)]: Miscellaneous

\section{INTRODUCTION}

Graphic designs, such as diagrams and maps, support a user's efforts to process sensory data, reason about it, solve problems, and identify patterns [23]. A well-designed visualization accounts for both the complex preattentive and cognitive processes triggered when a user views it. The balance of visual elements can dramatically affect the user's comprehension of the information presented. As a result of separable and integral feature relationships, using several elements in conjunction can amplify or dampen a single element's effect [13]. Understanding the relations among preattentive processes, cognitive processes, and visual interfaces is a longstanding goal of the visual analytics community, but evaluating interfaces in this context is difficult for many reasons. One such reason is the problem of individual differences.

As visualizations are meant to support complex thinking, they may be more sensitive to nuances of an individual user's cognitive style than other types of interfaces. This would explain findings that

*e-mail: cziemki@cs.brown.edu

†e-mail: rcrous01@cs.tufts.edu

†e-mail:yauillaa2@mailbox.winthrop.edu

$\S$ e-mail:sarasu@cs.tufts.edu

Ile-mail: ribarsky@uncc.edu

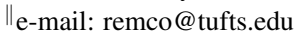

show significant differences in visualization performance based on personality. Earlier work has found an effect from the personality dimension known as "locus of control" $[10,11]$. This dimension measures a person's tendency to see herself as either shaped by or in control of external events. While the effects of individual differences such as locus of control have been observed, an explanation for why those differences arise remains elusive. Early work in this area employed real-world data exploration systems that differed on many dimensions, including the use of color, labeling, interaction, and layout style. Any of these variables may contribute to the effect of locus of control, making it difficult to directly apply the findings to visualization design for different user groups.

We propose that layout style is the key variable that determines the interaction between locus of control and compatibility with different system designs. Our definition of layout encompasses any differences in the spatial arrangement and presentation of marks in a visualization. This is to be distinguished from differences in the visual encoding, that is, how individual data variables are mapped to individual graphical variables, such as color or size ${ }^{1}$.

Our hypothesis is that individual differences based on locus of control are more affected by layout in this sense than by visual encoding or interaction style. This hypothesis is based on the idea that locus of control can provide insight into a user's tendency to rely on the external representations that a visualization employs. The overall arrangement of visual elements in a layout is more likely to directly affect the nature of this external representation than surface qualities such as color and shape, or more intangible qualities such as interaction style.

\subsection{Contributions}

This paper makes a number of contributions to our understanding of how personality factors affect how people use visualizations.

- First, we present experimental evidence showing that visual layout is a key factor in previous findings in individual differences. Our findings expand on previous work by showing that the effect of locus of control can still be found when restricting visualization differences to layout factors.

- Second, we use these findings to argue for a model of visualization use based on a user's adoption of external representations. Our findings suggest that locus of control affects the use of different visualization types by affecting a user's willingness to adapt to a novel externalization of information. This framework places these findings in the context of external representation as a model for visualization use.

We evaluate our hypothesis in a study with 240 online subjects who varied in their locus of control. Participants are presented with four variations of a hierarchy visualization showing phylogenetic data (Figure 1). These designs include a view that employs a list-like organizational structure (V1), a view that presents the hierarchy in

\footnotetext{
${ }^{1}$ In cases where a variable is directly mapped to spatial position, as in a scatterplot, that aspect of spatial arrangement is of course part of the encoding. No such mapping existed in the visualizations we study.
} 
a strong containment metaphor (V4), and two designs that fall in between these extremes (V2 and V3). We hypothesize that users with a more external locus of control are more willing to adapt their thinking to unfamiliar visual metaphors than those with an internal locus of control. We show how locus of control can predict performance on inferential task questions using these interface designs. Specifically, we test the hypothesis that an individual with a more internal locus of control will show a performance decrease when using layouts with a strong containment metaphor, while those with a more external locus of control will not show this decrease. Our findings provide evidence for the externalization model of visualization use and can inform the design of visualization interfaces adapted to an individual's needs.

\section{Related Work}

There is a substantial history of research in how individual differences affect interface use in the broader human-computer interaction field, reviewed by Dillon and Watson [6]. A subset of this research focuses specifically on individual differences in visualization, driven by the belief that a reliance on visual and spatial cognitive abilities makes individual differences a potentially greater factor in this field. We review findings on how cognitive differences affect visualization use and discuss why locus of control is a potentially significant factor.

\subsection{Individual Differences and Visualization}

Much of the existing work in the visualization field on the impact of individual differences has focused on the influence of perceptual ability on visualization use. Conati and Maclaren [5] find that a user's perceptual speed predicts whether a star graph or heatmap will be most effective. Similarly, Allen [1] finds a role for perceptual speed and spatial scanning ability in search performance. However, he found that users do not tend to optimize their visualization use for greater search efficiency, and proposes the development of user models to automatically guide users toward optimal strategies. This work shows that perceptual abilities can affect task performance, but suggests that the connection between these abilities and a user's cognitive strategies may be less direct.

Taking a more cognitive perspective, Chen [4] found no effect of spatial ability on participants' search performance in a visualization of paper citation links. Cognitive factors did, however, play a role in users' subjective feedback; users with high associative memory rated the interface higher on usefulness, and users with high spatial ability rated the tasks higher on familiarity. Chen goes on to suggest that users with varying cognitive abilities may be more or less likely to impose certain kinds of mental models on data.

A related finding comes from Ziemkiewicz and Kosara [31], who studied the role of personality and spatial ability in a user's ease in switching between incompatible visual metaphors in tree visualization use. They found that users with high spatial visualization ability and a high score on the Openness scale of the Big Five Personality Inventory [8] were more adept at answering questions despite a verbal metaphor that conflicted with the visual metaphor of the visualization they were viewing. Both this work and Chen's suggest that users with different cognitive styles may be more or less comfortable with adapting their thinking to an external representation.

Tversky et al. [26, 27, 15] have studied how individual differences in ability impact the extraction of structure and function from diagrams. They found that participants with high ability form mental models integrating structure and function, while those with low ability form models dominated by structure. They suggest that structural diagrams designed for the latter group can be annotated with functional information for improved comprehension [28].

These findings have begun to build the case that some individual differences in visual analytics can be attributed to a complex interaction between the mental model suggested by a visual layout

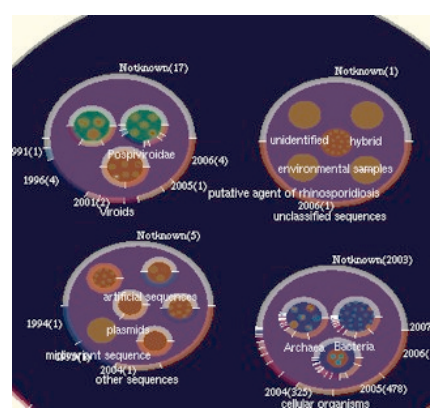

(a) GVis

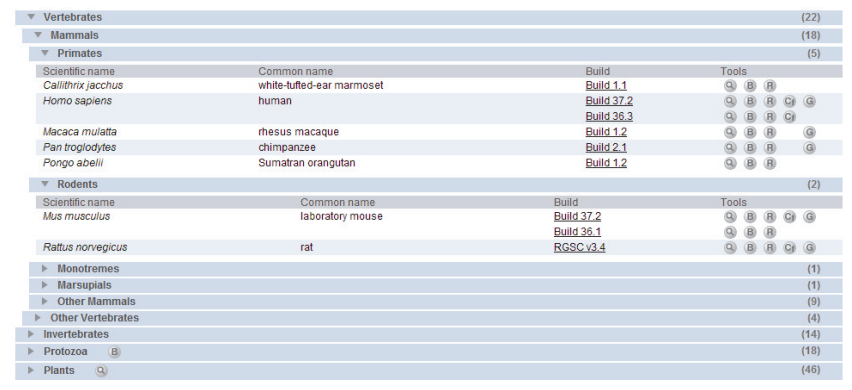

(b) NCBI Map Viewer

Figure 2: The two interfaces used in Green et al.'s study [10] of personality differences in visual analytics use.

and the user's own cognitive style. However, most previous studies that show an effect of individual differences either examine a single system or compare two or more systems with numerous differences. In order to apply knowledge of individual differences to visualization design, it is necessary to make a clearer connection between personality groups and exactly which factors lead them to better performance with one visualization over another. An area in which we argue that such a connection can be found is in locus of control and visual analytics performance.

\subsection{Locus of Control}

Much of the direct background for this work comes from Green et al.'s research $[10,11]$ on personality factors in visual analytics use. Their work found effects on interface performance from three psychometric measures: locus of control (LOC), neuroticism, and extraversion. Locus of control [24] measures the degree to which a person sees herself as in control of events (internal LOC) as opposed to seeing her fate as controlled by outside events (external LOC).

Locus of control has been associated with a number of significant practical outcomes. People with a more internal LOC tend to be more effective at work [17] and on many academic measures [7], and are more capable of coping with stress [2]. Locus of control in an academic setting is also associated with different learning styles. Cassidy and Eachus [3] found a positive correlation between external LOC beliefs and a tendency to use "surface learning" approaches, where the student attempts to fit the demands of an assignment instead of "deep learning," which focuses on the underlying principles being taught. Likewise, internal LOC students were more likely to use a deep learning approach. Such findings suggest the possibility that locus of control is related to how a user approaches problem-solving tasks in a novel setting, which may explain why it influences use of a novel visualization technique.

Green et al.'s work studied the effect of locus of control and other personality dimensions on both procedural and inferential learning in a GVis, a visual analytics interface (Figure 2(a)) versus NCBI 


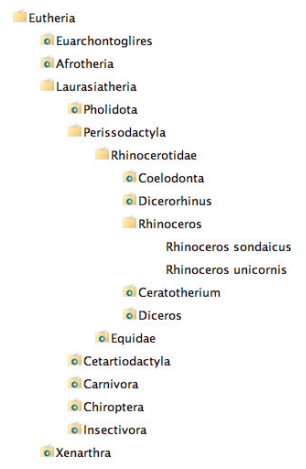

(a) V1: Basic Tree View

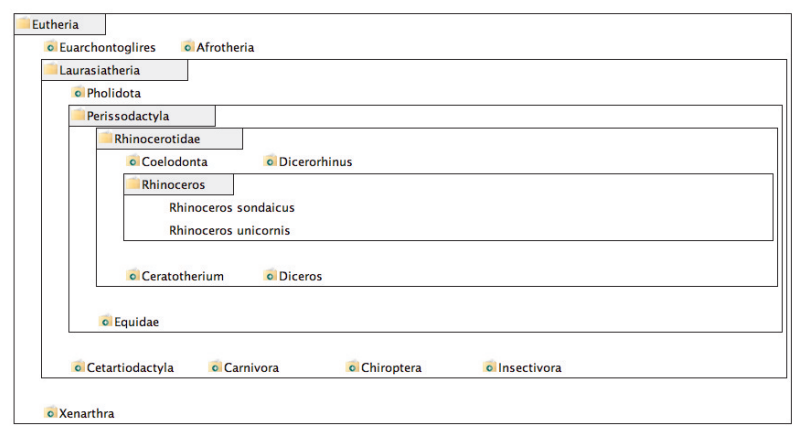

(c) V3: Indented Boxes View

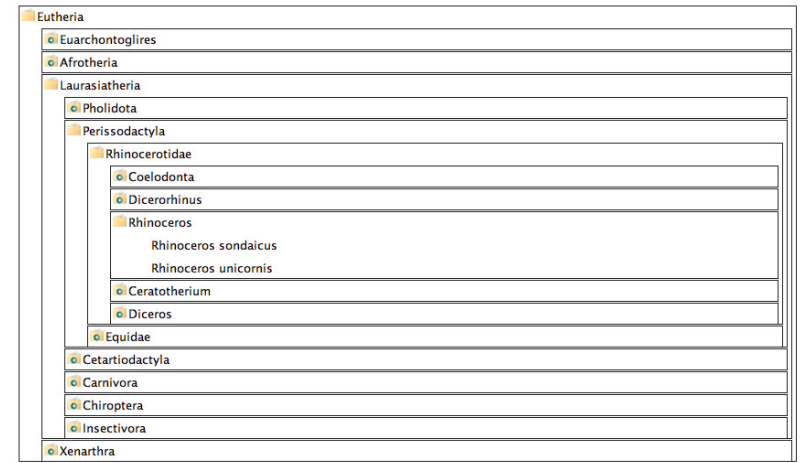

(b) V2: Bordered Tree View

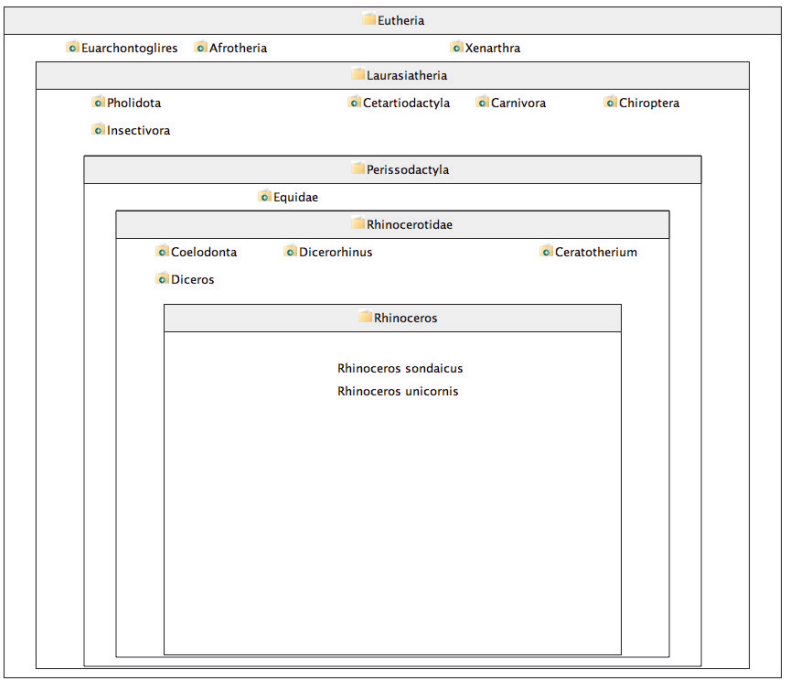

(d) V4: Nested Boxes View

Figure 1: The four visualizations used in the study. Each view is showing the same portion of one of the phylogenetic tree datasets.

Map Viewer, a more traditional web interface (Figure 2(b)). The procedural tasks they studied involved searching for a specific piece of information in a genomic database, while inferential tasks were those in which a user had to make a more open-ended comparison between two items. In both cases, the tasks were prompted by questions of the kind found in a typical usability study. The findings from both experiments suggested that the web table interface was more conducive to answering procedural questions and the findings from their first experiment suggest that the visual interface is more conducive to answering inferential questions.

In the first experiment, they found that participants with an external locus of control completed inferential tasks more quickly than those with an internal locus. This effect was more pronounced in GVis. In the second experiment, they studied only procedural tasks and found that, in contrast to inferential tasks, those with an internal locus of control completed procedural tasks more quickly.

In these studies, there were differences not only between interface structures but also between their interaction techniques. For example, GVis used a drilling-down zooming technique while Map Viewer used a menu driven interaction technique. In our work, we build upon these findings by seeking to isolate the factors of the interface structure that cause the different effects between those with an internal or external locus of control.

The amount of data we have about how different user types react to different interfaces is rapidly increasing. Our goal as visualization researchers must now be to make sense of this data within the context of models of the user. In order to apply this knowledge to improving design, we need to know not just what differences exist between users, but why. This work is an attempt to answer that question in relation to Green et al.'s findings on personality and interface design.

\section{HyPOTHESIS}

Research by Green et al. [10, 11] suggests that locus of control influences an individual's use of a complex visualization system. However, it seems counterintuitive that a personality trait with no known connection to visual or spatial ability should have any consistent influence over such a complex relationship. We propose that rather than an interaction between between locus of control and specific complex visualizations, the observed pattern may in fact be a correlation between locus of control and visual layout.

For example, consider the nested circles used in the GVis system from work by Green et al. [10, 11]; these structural elements are visually dominant due to their unusual shape and large size with respect to the surrounding textual elements. In contrast, Map Viewer uses a more subtle indentation-based structural expression which is dominated by text. Apart from using different visual encodings and interaction styles, these two designs represent significantly dif- 
ferent visual layouts of the same underlying data. Exploring how those layouts differ may help explain why locus of control interacts significantly with them.

To more closely investigate this correlation, we conduct a comparable study in which the test visualizations are more tightly controlled. We restrict the variation between our four test interfaces to visual layout style, holding interaction metaphor and visual encoding consistent across all interfaces. We hypothesize that, even under this simplified setting, participants with a more internal LOC will have difficulty with layouts that depend on a strong containment metaphor, while participants with a more external LOC will show a greater willingness to adapt to a variety of visual layouts.

\section{EXPERIMENT}

To test this hypothesis, we performed a user study in which participants were asked to answer search and inferential questions about data in four simple hierarchy visualizations (Figure 1). Like Green et al., we measured personality traits of the participants beforehand in order to test whether Locus of Control affects a participant's ability to use these visualizations. The four views were designed to express an increasingly visually explicit containment metaphor for the hierarchy, ranging from a list-like view that only used indentation to show hierarchical structure to a view that used large nested rectangles.

Green et al. [10] used two real-world systems in their work, which has the benefit of providing a realistic testing environment. However, this also makes it difficult to isolate exactly which aspects of the two designs prompted the differing user behavior they found. As the long-term goal of this work is to assist designers in choosing how to display information for varying user types, knowing exactly which elements of the design should be altered is vitally important. Therefore, our intention in designing the visualizations used in our study was, as much as possible, to isolate the factor of layout style which we hypothesized to be the key to these differences.

Before viewing the data, each participant was given a personality test, including the Locus of Control Inventory [24]. Each participant was then presented with a series of tasks to perform on each visualization. The order in which the visualizations were presented was randomized, and the user's ability to successfully accomplish tasks using each visualization was then recorded.

\subsection{Participants}

We recruited 240 participants over Amazon's Mechanical Turk service. Altogether, it took approximately two days to collect data from all participants. Mechanical Turk is an online job market in which people can be recruited for brief tasks and paid for their efforts. This service has become increasingly popular for use in online experiments, as a large number of relatively diverse participants can be processed very quickly [14]. Reservations remain about using Mechanical Turk among the human-computer interaction and visualization communities. However, since Mechanical Turk helps correct for a number of the traditional limitations of online studies, such as the possibility of vote flooding and the lack of incentive for completion [19], it is gradually becoming more accepted as a user study platform. It can be particularly useful in studies such as this, in which there is a ground truth by which to measure results [18] and the possibility of incentivizing accurate responses through bonuses [19].

That said, some limitations remain with interpreting online studies in general. Chief among these is environmental control. In an online study, it is impossible to know whether a participant's environment is noisy or distracting, or whether the participant is doing something else while performing the study. While these limitations should be kept in mind, this is a study with a clear ground truth and a task that should not be strongly affected by varying computing setups. In addition, our task questions were designed not to be answerable using a search engine. Under these circumstances, we argue that the advantages of using Mechanical Turk to obtain a high number of users outweigh the potential issues.

Of the 240 participants, four did not report their age or gender. Of the rest, there were 124 males and 112 females. Self-reported age ranged from 18 to 62 , with a mean of $26.7(\sigma=9.5)$. Our participants reported an average Locus of Control score of 3.61 $(\sigma=.59)$. This is slightly lower than scores reported in other publications that use this particular scale $[12,20]$. For example, Lapierre and Allen [20] find a mean Locus of Control of $4.03(\sigma=.61)$ for a participant pool of 205 employees in various professions. This difference may reflect the broader demographics of Mechanical Turk workers versus participants in traditional psychological studies. Locus of control in particular is often studied in the context of work or education, meaning the participants in these studies may have different educational or economic backgrounds than the general population. However, as we did not collect such demographics from our participants, we can only speculate on this point.

\subsection{Materials}

Participants were initially given two questionnaires to measure the aspects of their personality which are relevant to our hypotheses: a scale to measure the Big Five personality dimensions of Extraversion and Neuroticism and a Locus of Control scale to measure the degree to which they see themselves as in control of or controlled by external events. Both scales were taken from the International Personality Inventory Pool [9] and were combined to form a 40question survey. Neuroticism and Extraversion were included for comparison with Green et al.'s results, but are not the focus of the current analysis.

Green et al.'s study used two fully functional data exploration systems with many differences between them. For our study, we wish to isolate as much as possible the variable of layout style, and and so we created a set of four very specific visualizations (Figure 1). The first of these, V1 (Figure 1(a)) displays a tree in a simplified Windows Explorer style, using only indentation to indicate hierarchical relationships. This is representationally similar to the webpage organization used by Map Viewer in Green et al.'s work. The fourth view, V4 (Figure 1(d)) uses a nested boxes display that relies heavily on the visual metaphor of hierarchy as containment [30]. Although it uses rectangles rather than circles and a very different interaction style, this view is representationally similar to the nested bubbles of the GVis visual analytics system.

Between these two extremes, we designed two intermediate views. V2 (Figure 1(b)) is very similar to the indentation style of $\mathrm{V} 1$, but adds borders around the tree nodes to suggest a containment metaphor. V3 (Figure 1(c)) breaks the strictly vertical layout style used in V1 in favor of a horizontal layout closer to that used in V4, but still employs indentation to organize the levels of the hierarchy. These views are intended to provide cases that interpolate between the two layout styles used in Green et al.

Our use of such simplified views may raise the concern that our results do not directly apply to more realistic visual analytics scenarios. However, since we are partially attempting to replicate results from a study that employed real-world analysis systems, we feel this approach is complementary to previous work. If our results are similar to those found by Green et al, it would demonstrate that this simplification still maintains the important differences between the systems used in their study.

Furthermore, if a trend can be found to increase from V1 to V4, it would show that our intermediate views do indeed capture the major differences between the two views. Since the intermediate views primarily differ in the degree to which they express either a list-like or containment metaphor, this could support our argument that the finding is largely based on the different user groups' willingness to adapt to one metaphor over the other. That said, layout 


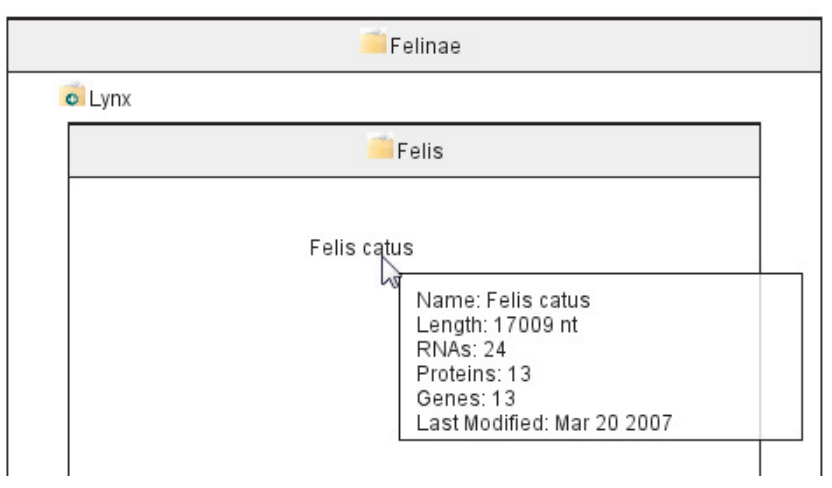

Figure 3: Tooltip showing species-level data.

is a complex factor that by nature is made up of many dimensions. While this study design is intended to keep these layout differences as controlled as possible, there are like other aspects of the layout, such as data-ink ratio or size of visual elements, which cannot be entirely ruled out as factors. Nonetheless, this study design can at least test whether layout factors in general can lead to a locus of control effect without differences in visual encoding or interaction.

Apart from these specific design differences, we attempted as much as possible to maintain consistency between the four views. They all use the same font size and folder icons. Each visualization also has the same interaction style, based on a collapsing folders metaphor such as that seen in a standard desktop file system. This may somewhat bias the results in favor of V1, which most closely resembles the interfaces used in file systems. Nonetheless, we argue that maintaining interaction consistency is important enough for isolating design factors that this is worth the tradeoff.

In addition, we implemented the restriction that only one subtree could be open at one time. If a user expanded one branch of the hierarchy and then attempted to expand a node in an unconnected branch, the first branch would automatically collapse. This was intended to keep the amount of potentially visible information consistent across the four views. Having several subtrees open is fairly easy in V1, which is purely vertical, but it would be difficult or impossible to open an infinite number of subtrees in V4 without making the lower-level nodes too small to display an entire label. Finally, to see genome data about individual species, the participant hovered the mouse over the species name to bring up a tooltip (Figure 3). Our goal with this simple if not necessarily intuitive interaction style was to keep the four views as consistent as possible except in how they visually organize the space.

The datasets presented in these four visualizations were four subsets of the full taxonomic tree from the National Center for Biotechnology Information's Genome database [22]. Each dataset consists of a phylogenetic tree where the leaf nodes are individual species. At the leaf level, there is some data on the genome mapping data available for that species, such as the date the entry was updated and the number of proteins and genes in the database. This is similar to the data used in Green et al., but does not include all the information found in the Map Viewer subset of the same database. In our case, we chose to show less data at the leaf level in order to present more data overall and more complex trees. The four datasets had, on average, 98.75 leaf nodes (i.e. individual species) and 114.75 non-leaf nodes in the phylogenetic tree. Details for each individual dataset are shown in Table 1. There was some variety in the branching factors and overall structure of the trees, although this was not carefully controlled for. However, since the datasets were ultimately balanced with respect to the view types, these differences should not affect our results substantially.

We considered the unfamiliarity of the datasets to be beneficial to
Table 1: The size of the four datasets used in the study.

\begin{tabular}{lll}
\hline Dataset Name & Number of Species & Non-leaf Nodes \\
\hline Amphibia & 92 & 94 \\
\hline Aves & 112 & 145 \\
\hline Eutheria & 92 & 94 \\
\hline Lepidosauria & 99 & 126 \\
\hline
\end{tabular}

our study, since we could trust that participants would need to consult the views in order to answer the task questions we presented them. Following Green et al. [11], these questions were divided into search tasks and inferential tasks. In both cases, tasks took the form of questions that participants were expected to consult the visualization to answer. This is similar to the methodology used in most visualization evaluation studies. These two question types are meant to represent simple data lookup and more complex analytical tasks, although they are simplified versions of the real-world versions of these tasks. We expect to see more differences in the inferential questions, since these are more likely to require understanding of the structure of a dataset rather than simple navigation ability.

The search questions asked the participants to find a single species within a classification that had a certain property. For example, "Within the classification 'Batrachuperus,' which species was most recently updated?" The verbal metaphor for tree structure used in these questions was varied between a levels and containment metaphor, to avoid any potential confound of metaphor compatibility [30]. Participants were asked to write the name of the species they found within a text field. The inferential questions were more open-ended, asking the participant to find a certain classification, then find another classification in another part of the taxonomy that had something in common with the first. For example:

Under "Anura," find the classification "Bufo" and note the subclasses it contains. There is another classification under "Mesobatrachi" that has something notable in common with "Bufo." Find that classification.

This was usually a similar pattern of names or numbers of species that fell under the classification. For example, the classification "Bufo" contained a classification also called "Bufo," and the correct answer was another classification that contained a child node with the same name as itself. Since correctness may be harder to judge in such a question with a free text response, participants in this case chose their response from a list of four multiple choice answers (as well as a "None of the Above" response). Each dataset was associated with two task questions: one search question and one inferential question. Participants therefore saw eight task questions altogether (Table 2).

\subsection{Procedure}

After selecting the study task from the Mechanical Turk website, participants were first asked to fill out the 40-question personality scale by rating each item in the scale from 1 (strongly disagree) to 5 (strongly agree). Once they were done, they read instructions on the main portion of the experiment.

The main portion of the experiment consisted of four sessions, one with each of the four views. The sequence in which participants saw these views was counterbalanced to prevent ordering effects. Each view was randomly associated with one of the four datasets. 
Table 2: The eight task questions seen in the study.

\begin{tabular}{lll}
\hline Dataset & Question Type & Question \\
\hline Amphibia & $\begin{array}{l}\text { search } \\
\text { inferential }\end{array}$ & $\begin{array}{l}\text { Within the classification "Batrachuperus," which species was most recently updated? } \\
\text { Under "Anura," find the classification "Bufo" and note the subclasses it contains. There is another classification } \\
\text { under "Mesobatrachia" that has something notable in common with "Bufo." Find that classification. }\end{array}$ \\
\hline Aves & $\begin{array}{l}\text { search } \\
\text { inferential }\end{array}$ & $\begin{array}{l}\text { Under the classification "Falco," find the species with a "Length" value over } 18000 . \\
\text { Looking in "Sphenisciformes," find the classification "Eudyptula" and note the species under it. Now look in } \\
\text { "Threskiornithidae" for a classification that has something notable in common with "Eudyptula." }\end{array}$ \\
\hline Eutheria & $\begin{array}{l}\text { search } \\
\text { inferential }\end{array}$ & $\begin{array}{l}\text { Within the classification "Tarsius," find the species which was most recently updated. } \\
\text { Under "Caniformia," find the classification "Canis" and note the subclasses and species it contains. Now find another } \\
\text { classification under "Ursidae" that has something notable in common with "Canis." }\end{array}$ \\
\hline Lepidosauria & $\begin{array}{l}\text { search } \\
\text { inferential }\end{array}$ & $\begin{array}{l}\text { Under the classification "Bipes," find the species with the lowest "Length" value. } \\
\text { Within "Scincomorpha," find the classification "Lacerta" and note the species under it. Now look in "Crotalinae" for } \\
\text { a classification which has something in common with "Lacerta." }\end{array}$ \\
\hline
\end{tabular}

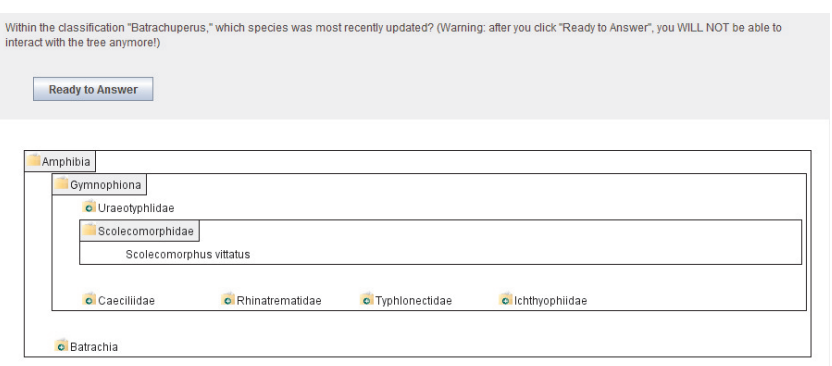

Figure 4: The basic study setup, showing a search task question.

Each participant saw all four datasets, but they appeared with equal probability in all four views.

When they first saw the visualization, participants were asked to familiarize themselves with it for as long as they wanted. When they were done, they clicked on a button labeled "Start" and were presented with the search question. They again had as long as they needed to interact with the visualization and find the answer. When they were satisfied with their answer, they clicked on a "Ready to Answer" button and were presented with a text field to fill in their answer. At this point, interaction with the visualization was locked. The purpose of this was to separate response time into the actual interaction time, without the time needed to type in the answer. A screen capture of this study setup is shown in Figure 4.

After answering the search question, the participant was presented with the inferential question for the same dataset. We presented the questions in this order since the inferential questions are naturally more difficult than the search tasks, and answering the search question would presumably give the user time to learn the basics of navigating the interface. The procedure for the inferential question was the same as for the search question, except that participants were presented with a set of multiple choice responses instead of a text field. These responses were presented in random order and were not visible during the interaction period.

Once both questions were answered, participants were shown a brief four-question preference survey on how much they liked the visualization. This survey included statements such as, "The system was easy to use," and "I enjoyed using this system." As in the personality scales, participants rated how much they agreed with each of these statements on a five-point scale. After completing all four visualization sessions, participants were asked to provide their age, gender, and any comments about the study in a form on the
Mechanical Turk site.

We measured the time a participant took in their initial training period, the time taken in the interaction period, and the time taken to record their response, as well as whether their response was correct or incorrect. In addition, we calculated each participant's Locus of Control based on their personality scale responses, and averaged their preference survey responses to generate a Preference Score for each of the visualizations they used.

\section{Results}

Our task questions proved to be quite difficult, with an overall accuracy of $68.6 \%$ correct responses on search tasks and $47.1 \%$ on inferential tasks. This difficulty, and the large amount of time spent interacting with the views, should be kept in mind when interpreting the following results. Across all participants and question types, no view condition was more or less difficult in terms of accuracy $\left(\chi^{2}(3, N=1919)=3.7, p=.29\right)$ or correct response time $(F(3,1109)=.57, p=.63)$. As our primary interest is in how these results varied with a participant's personality scores, further analysis focuses on participants grouped by personality type. Generally, we found support for our hypothesis that participants with a more internal LOC would have more difficulty with views more similar to V4. While we found that participants with a more external LOC did perform very well with V4, we did not find a corresponding trend in which they performed more poorly on views similar to V1.

\subsection{Effects of Locus of Control}

We initially divided participants into three groups based on their score on the Locus of Control scale. Participants with a score lower than one standard deviation from the mean (i.e. less than 3.01) were classified as external LOC users. Those with a score greater than one standard deviation from the mean (i.e. greater than 4.21) were classified as internal LOC users. The rest were classified as average LOC users.

The independent variables we analyzed to test our hypothesis were Question Type (search task versus inferential task), View Condition (V1, V2, V3, or V4), and LOC Group (external, average, or internal). Our overall model was therefore a $2 \times 4 \times 3$ Univariate Analysis of Variance (ANOVA). This overall model was significant for the variable of Correct Response Time $(F(23,1109)=10.67$, $p<.01)$. Correct Response Time only included correct responses, and represents only the time that a participant spent interacting with the view, not the time spent writing or choosing her response.

In addition, there were significant main effects of question type $(F(1,1109)=158.86, p<.01)$ and Locus of Control $(F(2,1109)=$ $5.38, p<.01)$. Unsurprisingly, search questions were answered 


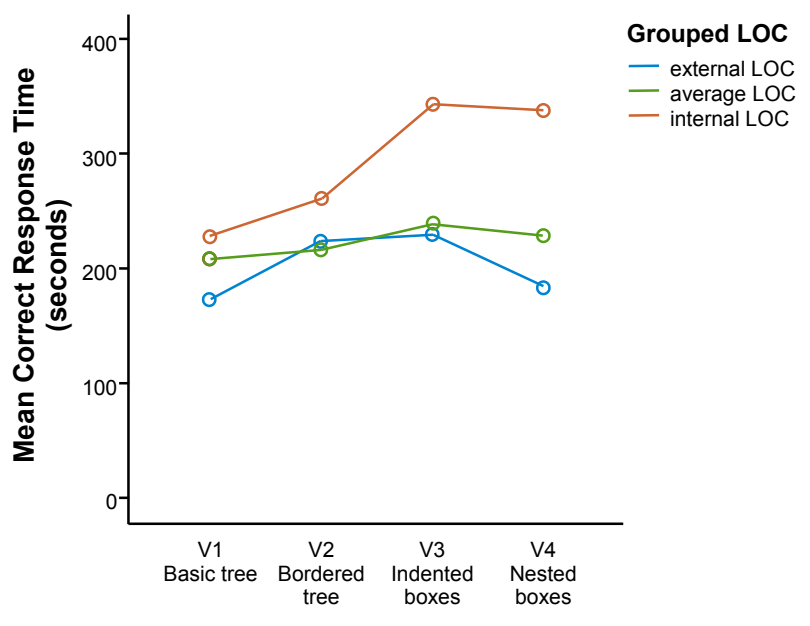

Figure 5: Response time for correct answers only across the four view conditions and three Locus of Control (LOC) groups. Participants with a highly internal LOC, who see themselves as in control of external events, were much slower than other participants at answering question in V4, a visualization that uses a strong nested-boxes visual metaphor. Participants with a highly external LOC, who see themselves as controlled by outside events, are relatively more likely to perform quickly on V4.

faster than the more difficult inferential questions. Overall, internal LOC participants were slower at answering questions correctly $(M=183.4$ seconds, $\sigma=11.6)$ than external LOC participants $(M=147.9$ seconds, $\sigma=10.3)$. There was also a significant interaction between view condition and question type $(F(3,1109)=3.01, p<.05)$ and between question type and LOC group $(F(2,1086)=5.93, p<.01)$. The reason for the latter is that internal LOC participants are much slower than the others on inferential tasks, but are the same speed when answering search tasks. The source of the interaction between view and question type is that, while inferential questions were answered more slowly than search questions in all views, this difference was significantly larger in V3. For inferential questions, V3 produced the slowest response times of all views, while it produced the fastest for search tasks.

The results relevant to our main hypothesis are summarized in Figure 5. We found that participants with an external LOC answer questions in V4 (the nested boxes) faster than other participants, although they answer questions in V1 faster as well. Internal LOC participants show a clear trend of slower performance from V1 to V4, although there is no distinction for these participants between V3 and V4. Average LOC participants show no response time difference between the four views.

In addition, we found that raw Locus of Control score correlates significantly with correct response time on nested boxes but no other condition $(r(104)=.23, p<.05)$. For search tasks, there was no significant response time difference for any participants between the four views.

To test overall accuracy, we used a Pearson's chi-square test on correctness and grouped Locus of Control. Participants with an external LOC answered more questions correctly overall $\left(\chi^{2}(2, N=\right.$ $1919)=7.7, p<.05)$, possibly due to their better performance on conditions V3 and V4. These results are summarized in Figure 6.

\subsection{Other Findings}

In addition to the personality variables that we measured, we analyzed our results based on the demographics and preference in-

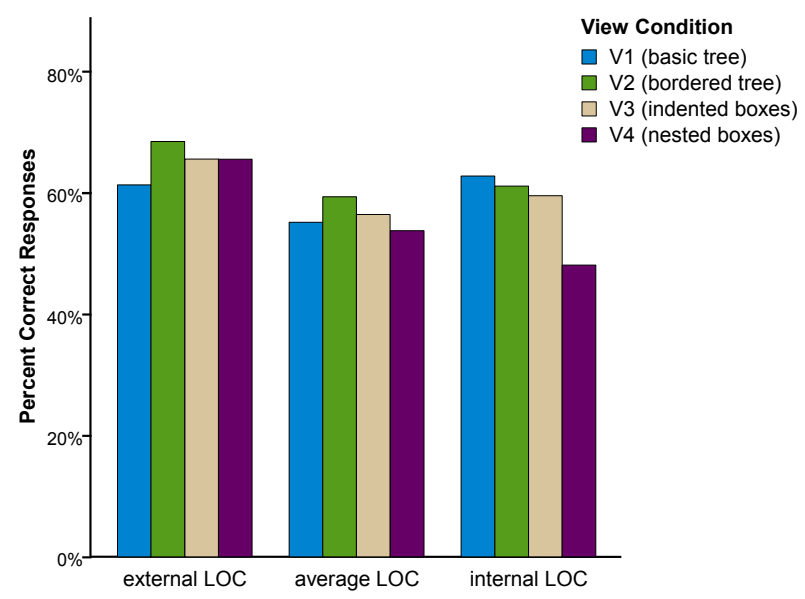

Figure 6: Percentage of correct answers across the four view conditions for participants grouped by their Locus of Control score. Participants with a more external locus of control were more accurate overall, while the other groups performed poorly with V4.

formation we collected from participants. According to a repeated measures ANOVA on the preference scores for each visualization, $\mathrm{V} 1$ and V2 were rated significantly more positively than V3 and V4 $(F(3)=15.09, p<.001)$. These preference ratings displayed no significant correlation with accuracy or response time. There was also no relationship between locus of control and preference ratings for any of the four views.

Female participants answered more questions correctly than male participants $(t(231)=-2.31, p<.05)$. We found that the age of participants correlated positively with overall response time $(r(1893)=.13, p<.001)$, so that older participants took more time interacting with the interfaces. Age had no effect, however, on accuracy, and did not correlate with any of the personality measures.

In the following sections, we will discuss these implications of these findings for our hypothesis and for visualization design in general.

\section{General Discussion}

In general, we replicated previously reported effects of locus of control in a simplified design that isolates the factor of overall layout style. This supports our main hypothesis that the significant factor of system design that interacts with locus of control is the way that visual elements are spatially arranged and presented, rather than interaction or visual encoding. This clarifies the earlier findings and points towards a potential explanation for this effect rooted in the relationship between locus of control and use of external representations.

\subsection{Replication and Expansion of Previous Findings}

Our results replicated those of Green et al. [10,11] in some cases but not completely. Error rates across the two experimental designs are not directly comparable, as in their case, participants were allowed to try as many times as needed to answer a question correctly, with each mistake recorded as an error. However, in both our study and theirs, we found that participants made more errors overall in the containment-metaphor visualization than in the more list-like view (V1 in our case, Map Viewer in theirs). More significantly, in both our work and theirs, participants with an external LOC responded faster to inferential questions than did participants with an internal LOC, particularly in a visualization with a containmentbased visual metaphor (V4 in our case, GVis in theirs). 
Together, these two sets of findings provide evidence that the effect of locus of control on visualization use is a robust one. Users with either an internal or external locus of control show performance differences in general on data exploration tasks, and additionally, each group performs better with different visualization styles. This suggests that locus of control is a variable that merits further study, and that personality differences are a valuable topic of research in visualization.

In general, we did not replicate their findings on search question response times. They found that internal LOC participants responded faster to search questions in GVis. However, their completion times for these tasks included any incorrect responses and subsequent guesses made by participants, so for these questions our response times are not directly comparable. This was not the case for the inferential questions, where they recorded only the time to make a single response.

Since our search questions were relatively difficult, it is unlikely that the lack of an effect in these questions is only a matter of performance differences not appearing in easier tasks. We speculate that the inferential questions forced the users to think in terms of the structure of the data to a greater degree. The search questions may have simply measured a participant's ability to navigate the interface quickly, while the inferential questions asked them to characterize parts of the data in an open-ended fashion. Participants may have interpreted these questions in a variety of ways, allowing the structural elements of the visualization design to play a greater role in their thought process.

Although external LOC participants were faster than other participant groups at answering questions in V4, they were equally fast in V1. This finding does not fit our original hypothesis that internal and external participants prefer different types of visual layouts. It may be that the very high familiarity of tree menus like V1 created a training effect that caused it to break the overall pattern. However, given the evidence, we cannot conclude that external participants perform better with containment views than with list-like views. Rather than a clear trend of group preference, a better interpretation of our results may be that external LOC participants are generally better able to answer inferential types of questions using unusual visualization layouts. An experiment that removes the potential confound of a highly familiar view would be needed to test which interpretation is better supported.

Familiarity may also explain the higher preference scores across all participants for V1 and V2, although it is interesting that there was no correspondence between preference and performance. It is also possible that this lack of a relationship may reflect the fact that our participants were paid a bonus for correct responses, and therefore had an incentive to perform well despite disliking the interface. In any case, people may have felt that V 3 and V4 were especially confusing due to their unusual appearance, but they were just as capable of answering questions with these interfaces.

\subsection{Locus of Control and External Representations}

By isolating the variable of layout style in this study, we have shown that simplified layout changes alone can produce locus of control effects similar to those found in a more realistic user study. This provides evidence that layout is a key factor in mediating this effect, independent of the effects of interaction style, visual encoding, and general differences between traditional interfaces and visual analytic systems. Although the current work does not directly support a causal explanation, we can speculate on why the different layouts we designed interacted with participant locus of control the way they did. We argue that the explanation for the behavior we observed is that participants with an external locus of control are willing to adapt more readily to visualization styles that employ a strong structural metaphor.

The four views in our study range from one (V1) which is dom- inated by white space and uses subtle visual organization to one (V4) which is dominated by screen elements expressing a visual metaphor of nested boxes. We argue that these views essentially represent a progression from visually implicit hierarchical structure to structure made visually explicit through layout and the use of lines and fills. V1 (Figure 1(a)) uses only a single dimension of spatial organization (horizontal indentation) and makes little distinction between leaf nodes and parent nodes. These factors make the hierarchical structure of the data less explicit. For example, two sibling nodes (such as Rhinocerotidae and Equidae in Figure 1(a)) can be visually separated to the point that their relationship is not immediately obvious. Because of this, we argue that V1 is the least structurally dominant of the four views.

After V1, each visualization in the sequence adds at least one visual element used to draw attention to the hierarchical structure of the data. The borders in V2 (Figure 1(b)) slightly emphasize the nesting of child nodes within parent nodes; for example, Rhinoceros and its two children are clearly grouped. V3 (Figure 1(c)) takes this further by highlighting the names of parent nodes and arranging child nodes horizontally to emphasize a spatial metaphor of containment. Finally, V4 (Figure 1(d)) gives the parent node a strong visual emphasis with centering and a title bar and collects the child nodes together at the top of the parent to make sibling relationships obvious. This is the least "list-like" of the four views and uses the most "ink" and screen space to express hierarchical relationships.

The connection between this use of explicit visual structure and locus of control may be explained by the body of thought that views visualization as an external mental representation. The field of distributed cognition [16] sees mental processes such as problem solving and memory as relying not only on knowledge stored in the mind, but also on knowledge stored in a person's environment, in the form of physical objects, information artifacts, and other people. Applying this perspective to visualization, Liu et al. [21] have argued that a primary benefit of visualization is the externalization of information. Externalization makes problem-solving more efficient and accurate by substituting quicker perceptual processes for cognitive processing of information. Furthermore, studies have shown that the form of these representations can influence problemsolving strategies.

Users with an external locus of control are those who expect the outside world to dominate their fate. Taking a distributed cognition approach, it is possible that these users also rely more heavily on outside representations, rather than internal mental representations, when solving problems or making sense of information. Conversely, those with an internal locus of control may prefer to perform cognitive tasks more internally, relying less on external representations. In general, this makes people with an internal locus of control more adept at problem-solving and learning, as the extensive literature on locus of control has shown. However, it is possible that this same tendency to rely on internal representations may make it more difficult to use the complex external representations found in a visual analytics system.

If this is indeed the case, a visualization with a highly explicit and unfamiliar visual structure may be more jarring for an internal LOC user. Someone with an external locus of control may be more willing by nature to adapt her thinking to the external representation, while the user with an internal locus may be going through a more difficult process of fitting the external representation to her own ideas of what the data is like. Cassidy and Eachus's work [3], discussed in Section 2.2, implies that the "surface learning" approach taken by external LOC students is academically harmful, and based on well-established findings in locus of control research, this does seem to be the case in general. However, this very tendency to focus on surface structure may be beneficial in the context of learning a new visualization system. Our external LOC partici- 
pants were just as fast with a novel visualization as they were with the kind of indented list they see on their computer desktop every day. There may be other ways of interpreting these effects, and for now, this is a hypothesis for future research, not a firm conclusion. Nevertheless, this potential ability to make an advantage out of a personality style that is usually considered problematic suggests intriguing future directions in the application of visualization to learning.

Taken together with previous work, these findings contribute to the case for an externalization-based view of how people perform complex tasks with a visualization. Furthermore, they imply that this externalization process varies greatly between people and situations, which may be a significant factor in the difficulty of controlling and intepreting evaluations of visualization systems. We discuss the implications of these findings for design and evaluation in the following section.

\section{DESIGN IMPLICATIONS}

This study and others like it provide mounting evidence that personality and design style can have a significant effect on whether a user accepts a visualization design. It is possible that a user's personality can serve as shorthand for subtle cognitive style differences that are not easily measureable otherwise, but which gain importance in the exploratory context of visualization use. When we give users a novel visualization, we are essentially asking them to give up some control over their thinking processes. Some users will find this helpful, while others may find it a hindrance. We argue that a visualization designer should have a sense of how willing a given user will be to take on an external representation, and know how to design a visualization that makes it more or less difficult to ignore the structural aspects of that representation.

Based on our findings, a useful guideline for adaptation would be to increase the amount of explicit structure for users that might have a more external locus of control. Users with a very internal LOC will most likely perform best with a visualization style that uses simple spatial organization and minimal borders, outlines, and other grouping elements. In practice, this type of design may correspond to the maximized "data-ink ratio" argued for by Tufte [25]. External LOC users, on the other hand, may perform more efficiently with a visualization style that violates this classic guideline by including more non-functional elements such as borders, fills, and outlines to call attention to a specific information structure. In addition, this type of user may have an easier time working with visualizations that use a two-dimensional spatial layout to organize information.

Although it would usually be impractical to directly measure a user's locus of control and adapt the visualization accordingly, it is still possible to use this principle to guide design. Our perspective sees locus of control as predicting the degree to which a user will, by innate disposition, prefer her own internal mental models (internal LOC) versus being willing to adapt to an external representation (external LOC). This general principle, then, may apply in other situations where users are likely to prefer a pre-existing mental model or problem-solving process for reasons other than personality. Expert users, for example, may be more resistent to visualizations with highly explicit structure. Likewise, a user group with a highly standardized analysis process should be given visualizations with a low structural emphasis. A user group which is likely to approach a problem in a more exploratory mode may find it easier to work with a visualization that makes the structural organization of data more explicit.

Visualization, especially in the context of more complex, openended problems, involves explorations that can require search, organizing and filtering, inference-building, and iteration in combinations that cannot be predicted beforehand. The results presented in this paper and the design guidelines suggested will be important in the construction of effective visualization tools and methods for this open-ended cognitive process. In addition to telling us something about where adaptation to personality type can be significant, the results tell us where adaptation is less so, permitting the freer use of visual representations and interaction styles determined by other factors. All this will help lead to the development of a model of human reasoning in the presence of automated analysis, which is of central importance to the emerging field of visual analytics.

\section{Future WORK AND CONCLUSION}

In subsequent work, we hope to combine these findings with other work in individual differences to pursue more complete models of the visualization user. As Yi [29] has argued, major advances in understanding these individual differences must come from collecting knowledge across studies. This work is one attempt at doing so, and much more can be done to find commonalities among the measures and findings on how users adapt to visualizations.

In this paper, we have contributed findings on how users with different personality types react to varying layout styles used in a hierarchy visualization. We found evidence that systematic differences in layout style can indeed influence a user's response time and accuracy with different types of visualizations that are informationally equivalent but differ in layout. These findings seem to fit a pattern in which users with a more external locus of control are more efficient at using a visualization which uses a highly explicit visual metaphor than users with a more internal locus of control. We hope that these findings can serve as a step towards better understanding of why subtle differences between users' personality styles can have a surprising influence on visualization use.

\section{ACKNOWLEDGEMENTS}

The authors wish to thank Tera M. Green and Brian Fisher for their helpful discussions and collaboration. This material is based in part upon work supported by the International Program of the Department of Homeland Security under grant number 2009-ST108-000007. Any opinions, ndings, and conclusions or recommendations expressed in this material are those of the authors and do not necessarily reect the views of the Department of Homeland Security. This material is based in part upon work supported by the National Science Foundation under Grant No. CIF-B-195. Any opinions, findings, and conclusions or recommendations expressed in this material are those of the authors and do not necessarily reflect the views of the National Science Foundation.

\section{REFERENCES}

[1] B. Allen. Individual differences and the conundrums of user-centered design: Two experiments. Journal of the American Society for Information Science, 51(6):508-520, 2000.

[2] C. R. Anderson. Locus of control, coping behaviors, and performance in a stress setting: A longitudinal study. Journal of Applied Psychology, 62(4):446-451, 1977.

[3] S. Cassidy and P. Eachus. Learning style, academic belief systems, self-report student proficiency and academic achievement in higher education. Educational Psychology, 20(3):307-320, 2000.

[4] C. Chen. Individual differences in a spatial-semantic virtual environment. Journal of the American Society for Information Science, 51(6):529-542, 2000

[5] C. Conati and H. Maclaren. Exploring the role of individual differences in information visualization. In Advanced Visual Interfaces. ACM Press, 2008.

[6] A. Dillon and C. Watson. User analysis in HCI: The historical lesson from individual differences research. International Journal of HumanComputer Studies, 45:619-637, 1996.

[7] M. J. Findley and H. M. Cooper. Locus of control and academic achievement: A literature review. Journal of Personality and Social Psychology, 44(2):419-427, 1983. 
[8] L. R. Goldberg. An alternative "description of personality": The bigfive factor structure. Journal of Personality and Social Psychology, 59(6):1216-1229, 1990.

[9] L. R. Goldberg, J. A. Johnson, H. W. Eber, R. Hogan, M. C. Ashton, C. R. Cloninger, and H. C. Gough. The international personality item pool and the future of public-domain personality measures. Journal of Research in Personality, 40:84-96, 2006.

[10] T. M. Green and B. Fisher. Towards the personal equation of interaction: The impact of personality factors on visual analytics interface interaction. In IEEE Visual Analytics Science and Technology (VAST), 2010.

[11] T. M. Green, D. H. Jeong, and B. Fisher. Using personality factors to predict interface learning performance. In Hawaii International Conference on System Sciences 43, January 2010.

[12] S. Hamlyn-Wright, R. Draghi-Lorenz, and J. Ellis. Locus of control fails to mediate between stress and anxiety and depression in parents of children with a developmental disorder. Autism, 11(6):489-501, 2007.

[13] C. G. Healey. Perception in visualization. North Carolina State University, http://www.csc.ncsu.edu/faculty/healey/PP, Retrieved March 2011.

[14] J. Heer and M. Bostock. Crowdsourcing graphical perception: Using Mechanical Turk to assess visualization design. In Proceedings CHI, pages 203-212, 2010.

[15] J. Heiser and B. Tversky. Mental models of complex systems: Structure and function. http://wwwpsych.stanford.edu/simwbt/diagrams/papers/mental_models-heisertversky.pdf.

[16] J. Hollan, E. Hutchins, and D. Kirsh. Distributed cognition: Toward a new foundation for human-computer interaction research. $A C M$ Transactions on Computer-Human Interaction, 7(2):174-196, 2000.

[17] T. A. Judge and J. E. Bono. Relationship of core self-evaluations traits-self-esteem, generalized self-efficacy, locus of control, and emotional stability - with job satisfaction and job performance: A meta-analysis. Journal of Applied Psychology, 86(1):80-92, 2001.

[18] A. Kittur, E. H. Chi, and B. Suh. Crowdsourcing user studies with Mechanical Turk. In Proceedings CHI, pages 453-456, 2008.

[19] R. Kosara and C. Ziemkiewicz. Do Mechanical Turks dream of square pie charts? In BELIV Workshop, pages 373-382, 2010.

[20] L. M. Lapierre and T. D. Allen. Control at work, control at home, and planning behavior: Implications for workfamily conflict. Published online before print in Journal of Management, 2010.

[21] Z. Liu, N. Nersessian, and J. Stasko. Distributed cognition as a theoretical framework for information visualization. IEEE Transactions on Visualization and Computer Graphics, 14(6):1173-1180, 2008.

[22] National Center for Biotechnology Information. NCBI genome database. http://www.ncbi.nlm.nih.gov/genome/. Accessed 11/18/2010.

[23] S. E. Palmer. Vision science: Photons to phenomenology, volume 1. MIT Press., 1999.

[24] J. B. Rotter. Generalized expectancies for internal versus external control of reinforcement. Psychological Monographs, 80(609), 1966.

[25] E. R. Tufte. The Visual Display of Quantitative Information. Graphics Press, 1983.

[26] B. Tversky. Functional significance of visuospatial representations. In P. Shah and A. Miyake, editors, Handbook of higher-level visuospatial thinking, pages 1-34. Cambridge University Press, 2005.

[27] B. Tversky, M. Agrawala, J. Heiser, P. U. Lee, P. Hanrahan, D. Phan, C. Stolte, and M.-P. Daniele. Cognitive design principles: From cognitive models to computer models. In L. Magnani, editor, Model-based reasoning in science and engineering, pages 1-20. King's College, 2007.

[28] B. Tversky, J. Zacks, P. U. Lee, and J. Heiser. Lines, blobs, crosses, and arrows: Diagrammatic communication with schematic figures. In M. Anderson, P. Cheng, and V. Haarslev, editors, Theory and application of diagrams, pages 221-230. Springer, 2000.

[29] J. S. Yi. Implications of individual differences on evaluating information visualization techniques. In Proceedings of the BELIV Workshop, 2010.

[30] C. Ziemkiewicz and R. Kosara. The shaping of information by visual metaphors. IEEE Transactions on Visualization and Computer Graphics, 14(6):1269-1276, 2008.

[31] C. Ziemkiewicz and R. Kosara. Preconceptions and individual differences in understanding visual metaphors. Computer Graphics Forum, 28(3):911-918, 2009. Proceedings EuroVis. 